\title{
Morte Materna em Hospital Terciário do Rio Grande do Sul - Brasil: um Estudo de 20 Anos
}

\author{
Maternal Mortality at a Tertiary Hospital in Rio Grande do Sul - Brazil: a Twenty-year Study \\ José Geraldo Lopes Ramos, Sérgio Martins-Costa, Janete Vettorazzi-Stuczynski, Elisa Brietzke
}

\begin{abstract}
RESUM0
Objetivo: analisar os casos de morte materna ocorridos no Hospital de Clínicas de Porto Alegre (HCPA), hospital universitário de referência para gestação de alto risco no Rio Grande do Sul.

Métodos: realizamos estudo retrospectivo analisando os prontuários médicos das mulheres entre 10 e 49 anos que morreram no HCPA no periodo de 1980 a 1999. Foram analisadas apenas as mortes relacionadas a gestação e puerpério laté 365 dias após o término da gestação), independente do tipo e duração da gestação. As causas foram separadas em causas obstétricas diretas, obstétricas indiretas e causas não obstétricas.

Resultados: entre as causas obstétricas diretas (61,7\%), destacaram-se a hipertensão arterial (18,5\%), a infecção pós-cesariana (16\%) e o aborto séptico (12,3\%). Dentre as causas obstétricas indiretas (23,5\%), as mais prevalentes foram a cardiopatia (8,6\%), o figado gorduroso agudo (3,5\%) e o lúpus eritematoso sistêmico (2,5\%). Dentre as causas não obstétricas (15,0\%), destacam-se as neoplasias malignas $(7,4 \%)$ e a AIDS $(3,7 \%)$.

Conclusões: a prevalência das principais causas de morte materna não sofreu modificação nas últimas duas décadas, sendo que a principal causa continua sendo a hipertensão arterial. Também, há número significativo de mortes relacionadas à cesariana (relacionadas ao procedimento) e às infecções. Podemos concluir que a prevalência de causas obstétricas diretas aponta para a baixa capacidade de prevenção de morte materna no nosso sistema de saúde.
\end{abstract}

PALAVRAS-CHAVE: Morte materna. Hipertensão arterial. Infecção pós-cesariana. Gestação de alto risco. Aborto séptico. Cesariana.

\section{Introdução}

Um dos maiores desafios dos países não desenvolvidos é o de promover substancial diminuição da mortalidade materna. A comparação dos indicadores de mortalidade materna entre países desenvolvidos como Canadá e Estados Unidos, que

Serviço de Ginecologia e Obstetrícia do Hospital de Clínicas de Porto Alegre

Departamento de Ginecologia e Obstetrícia da Faculdade de Medicina da Universidade Federal do Rio Grande do Sul

Correspondência:

José Geraldo Lopes Ramos

Serviço de Ginecologia e Obstetrícia do Hospital de Clínicas

de Porto Alegre

Avenida Ramiro Barcelos, 2350, sala 1125

90035-000 - Porto Alegre - RS

Fax: (51) 3316-8148

e-mail: ramosjg@terra.com.br têm razão de mortalidade materna inferior a 9:100.000 nascidos vivos, e países como Bolívia, Peru e Brasil, com razões superiores a 100 mortes por 100.000 nascidos vivos, evidencia muito bem a disparidade entre estes dois blocos ${ }^{1}$. Entretanto, países não desenvolvidos que estabeleceram metas de política que garantem saúde para a sua população, como Chile, Cuba, Costa Rica e Uruguai, conseguiram diminuir suas taxas de mortalidade materna para menos de 40 óbitos por 100.000 nascidos vivos ${ }^{1}$.

O Brasil ainda não possui sistema de registros adequado para notificação e classificação da mortalidade materna, seja esta direta ou indire$\mathrm{ta}^{2}$. No Rio Grande do Sul há um esforço conjunto para o melhoramento do sistema de registros através do estabelecimento de comitês de mortalidade materna, o que vem melhorando a qualidade 
dos registros, especialmente na cidade de Porto Alegre. O estabelecimento de comitês de investigação de morte materna, funcionando tanto em âmbito regional (estados e municípios) como local (comitês hospitalares), tem sido um dos instrumentos mais eficazes para o reconhecimento da dimensão real da mortalidade materna e o estabelecimento de diretrizes específicas para a diminuição de sua ocorrência. Em um país como o Brasil, no qual a quase totalidade dos partos ocorre dentro do sistema hospitalar, a investigação dos óbitos de mulheres nos hospitais é o mais forte instrumento para erradicar a subnotificação e o sub-registro da mortalidade materna. Para tanto é necessário que os comitês de óbitos hospitalares investiguem todos os óbitos de mulheres em idade fértil e não somente as mortes ocorridas em pacientes internadas na maternidade, uma vez que, principalmente nos hospitais de nivel terciário de atenção de saúde, não é raro que mulheres são internadas com quadros graves diretamente nos centros de terapia intensiva. Estas mulheres freqüentemente recebem diagnósticos gerais e imprecisos, como sepse, coagulação vascular disseminada, choque e tantos outros, que são, na verdade, complicações de eventos obstétricos, como abortamento séptico, embolia amniótica, sindrome HELLP, etc. ${ }^{1}$. Portanto, a chave para a realização de estudos de base populacional fidedignos, é a análise preliminar feita em âmbito hospitalar com a divulgação dos coeficientes ou razões de mortes maternas hospitalares.

Com os objetivos de estabelecer estratégias de combate à mortalidade materna hospitalar e de fornecer dados fidedignos aos comitês municipais e estaduais de mortalidade materna, realizamos estudo retrospectivo com a análise de todos os casos de morte materna ocorridos no Hospital de Clínicas de Porto Alegre (HCPA), hospital universitário de referência para gestações de alto risco que funciona na capital do Estado do Rio Grande do Sul, o qual apresenta uma população com cerca de 10.000.000 habitantes.

\section{Pacientes e Métodos}

Realizamos estudo retrospectivo e descritivo, baseado na revisão e análise de registros em prontuários médicos. A população em estudo consistiu de pacientes do sexo feminino em idade fértil e que morreram no HCPA, no período entre 1980 e 1999. Todos os prontuários médicos de mulheres com registro de óbito no hospital durante o referido período foram avaliados, tendo sido destacados para análise inicial aqueles de mulheres com idade entre 10 e 49 anos. Foram detectados
1370 óbitos de mulheres e destes foram separados para estudo todos os casos relacionados com a gestação e o puerpério.

Utilizou-se, para fins deste estudo, a razão de mortalidade total, ou seja, os óbitos obstétricos diretos, os indiretos e a mortalidade materna tardia, ou seja, os óbitos ocorridos durante a gestação, o parto e o puerpério, estendendo-se este período até 365 dias após o término da gestação. As causas foram separadas em causas obstétricas diretas, obstétricas indiretas e causas não obstétricas, conforme sua correlação com doenças da gravidez, do parto ou do puerpério. Consideramos como morte por sepse as ocorridas em conseqüência à infecção em gestações após a $20^{\mathrm{a}}$ semana e antes do parto, independentemente do sítio inicial da infecção, e aborto séptico as ocorridas antes da $20^{\mathrm{a}}$ semana.

Os dados obtidos foram processados eletronicamente utilizando-se o programa Epi-Info versão 6.0 e aplicando-se os teste do $\chi^{2}$. As características populacionais foram apresentadas como médias, freqüências e desvio padrão. O presente estudo foi aprovado pela Comissão de Ética em Pesquisa do HCPA. Os princípios bioéticos de beneficência, nãomaleficência, justiça, confiabilidade, privacidade e veracidade foram respeitados.

\section{Resultados}

Entre 1980 e 1999 ocorreram 1544 óbitos de mulheres em idade reprodutiva; dessas, 81 estavam no período grávido-puerperal (até 365 dias após o parto), com média de idade de 28,5 anos em faixa de variação de 17 a 42anos. No mesmo período, ocorreram 63.002 nascimentos (nativivos), sendo que a razão de mortalidade materna total (incluindo-se os casos não obstétricos) foi de 129:100.000 nascidos vivos. Se analisarmos somente os casos com nascimento no HCPA, a razão situa-se em 90:100.000 nascidos vivos. Como para fins de cálculo da razão de mortalidade materna devem-se excluir os casos de morte materna não obstétrica ${ }^{1}$, obtém-se razão de mortalidade total (obstétricas diretas + obstétricas indiretas + não especificadas) de 109:100.000 nascidos vivos.

Quanto às características da população estudada, observamos que $77,3 \%$ eram de cor branca, 64,1\% tinham situação conjugal estável, 58,5\% tinham feito pelo menos uma consulta de pré-natal e $20,7 \%$ eram primigestas.

Quanto ao momento gestacional e à via de parto, dos 81 óbitos, 41 (50,0\%) ocorreram em pacientes submetidas à cesariana, $15(18,7 \%) \mathrm{em}$ pacientes que tiveram parto vaginal, $10(12,5 \%)$ 
quando a gravidez terminou em aborto, 14 (17,5\%) em gestantes durante o pré-natal e um $(1,2 \%)$ para o qual não foi possivel determinar o momento gestacional ou o tipo de parto (esse caso foi internado diretamente na unidade de tratamento intensivo em coma, onde não havia familiares ou registro do parto).

Entre os 41 casos de morte em pacientes submetidas à cesariana, 18 tiveram causas diretamente relacionadas com o ato operatório/anestésico, como infecção pós-cesariana (13 casos), acidente anestésico ( 3 casos), hemorragia (1 caso) e embolia por líquido amniótico (1 caso), ao passo que em 23 pacientes as causas de óbito não puderam ser imputadas ao ato cirúrgico.

Quando comparamos as mortes de pacientes com cesariana que foram relacionadas ao ato operatório e/ou anestésico com os óbitos relacionados ao parto transpélvico, pode-se observar risco relativo de morte associado ao procedimento cirúrgico 10 vezes maior em pacientes submetidas à cesariana (Tabela 1).

Tabela 1 - Risco relativo de morte materna conforme a via de parto nas pacientes que tiveram procedimento obstétrico realizado no HCPA (1980-1999).

\begin{tabular}{|c|c|c|c|}
\hline \multicolumn{2}{|c|}{$\begin{array}{l}\text { Cesariana } \\
(n=18.082)\end{array}$} & \multicolumn{2}{|c|}{$\begin{array}{l}\text { Parto vaginal } \\
(n=44.920)\end{array}$} \\
\hline Causa & $\mathrm{n}$ & Causa & $\mathrm{n}$ \\
\hline Infecção & 8 & Infecção & 1 \\
\hline Acidente anestésico & 3 & & \\
\hline Hemorragia & 1 & Hemorragia & 2 \\
\hline $\begin{array}{l}\text { Embolia por líquido } \\
\text { amniótico }\end{array}$ & 1 & & \\
\hline Total & 13 & & 3 \\
\hline Razão de mortalidade & $1: 1.390$ & & $1: 14.973$ \\
\hline
\end{tabular}

Risco relativo $=10,77(3,07-37,77) ; p=0,000003$

Entre as causas obstétricas diretas, que perfazem um total de 50 casos (62\%), a causa básica mais prevalente de óbito foi a hipertensão arterial, com 14 casos $(17,2 \%)$, seguida da infecção pós-cesariana com 13 casos (16\%) e do aborto séptico, com 10 casos (12,3\%). A sepse contribuiu com 2 casos $(2,5 \%)$. O acidente anestésico e a hemorragia puerperal, cada um com três casos $(3,7 \%)$, e a mola hidatidiforme, a corioamnionite, a embolia por líquido amniótico e a infecção de episiotomia, cada uma contribuindo com um caso $(1,2 \%)$, completam o quadro dos óbitos por causas obstétricas diretas. A razão de mortalidade materna para as causas obstétricas diretas foi de 79:100.000 nascidos vivos. Dentre as causas obstétricas indiretas, com um total de 19 casos $(23,5 \%)$, a cardiopa- tia foi a mais prevalente, com 7 mortes $(8,6 \%)$, seguida pelo fígado gorduroso agudo com três casos $(3,7 \%)$ e pelo lúpus eritematoso sistêmico com dois casos $(2,5 \%)$. O tétano, a colite pseudomembranosa, a infecção do trato urinário associada a insuficiência renal aguda, a broncopneumonia e a cetoacidose diabética, cada um com um caso $(1,2 \%$ cada), completam as causas indiretas (Tabela 2). Dentre as causas não obstétricas, que somam 12 casos (15\%), foram encontrados seis casos $(7,4 \%)$ de neoplasia maligna (1 câncer de mama, 1 câncer renal, 2 coriocarcinomas e 2 linfomas), três casos de AIDS (3,7\%), um caso de politraumatismo por acidente de trânsito $(1,2 \%)$, um caso de cetoacidose diabética e, em um caso, a causa da morte não pôde ser esclarecida.

Tabela 2 - Causas básicas de mortalidade materna no HCPA (1980-1999).

\begin{tabular}{lrr}
\hline & $\mathbf{n}$ & $\%$ \\
\hline Causas obstétricas diretas & & \\
Hipertensão associada à gestação & 14 & 17,28 \\
Infecção pós-cesárea & 13 & 16,04 \\
Aborto séptico & 10 & 12,34 \\
Acidente anestésico & 3 & 3,70 \\
Hemorragia puerperal & 3 & 3,70 \\
Sepse (parto pré-termo) & 2 & 2,47 \\
Outras causas & 4 & 4,92 \\
Subtotal & 50 & 61,7 \\
Causas obstétricas indiretas & & \\
Cardiopatia & 7 & 8,64 \\
Fígado gorduroso & 3 & 3,70 \\
Lúpus eritematoso sistêmico & 2 & 2,47 \\
Tromboembolismo venoso & 2 & 2,47 \\
Outras causas & 6 & 7,40 \\
Subtotal & 19 & 23,5 \\
Causas não obstétricas & 12 & 14,8 \\
Total & 81 & 100 \\
\hline
\end{tabular}

Dentre as causas infecciosas, que somam 32 dos 81 casos de morte $(39,5 \%)$, a infecção póscesariana mostrou-se a mais prevalente, com 13 casos $(40,6 \%)$, seguida do abortamento séptico com sete casos $(21,9 \%)$. As causas obstétricas indiretas encontradas foram as seguintes: infecção do trato urinário com insuficiência renal aguda, tétano, endocardite por listéria, colite pseudomembranosa e broncopneumonia, com um caso cada $(3,1 \%)$. Entre as causas infecciosas não obstétricas destaca-se a AIDS como responsável por três casos $(9,4 \%)$ de morte materna (Tabela 3$)$. 
Tabela 3 - Mortalidade materna por causas infecciosas no HCPA no período de 1980 a 1999.

\begin{tabular}{lrr} 
Causas infecciosas & $\mathbf{n}$ & $\%$ \\
\hline Obstétricas diretas & 13 & 40,6 \\
$\quad$ Infecção pós-cesárea & 7 & 21,9 \\
Aborto séptico & 1 & 3,1 \\
Infecção da episiotomia & 1 & 3,1 \\
Corioamnionite & 2 & 6,2 \\
Sepse (parto pré-termo) & 24 & 75,0 \\
Subtotal & & \\
Obstétricas indiretas & 1 & 3,1 \\
$\quad$ Infecção urinária/Insuficiência renal aguda & 1 & 3,1 \\
Tétano & 1 & 3,1 \\
Endocardite por listéria & 1 & 3,1 \\
Colite pseudomembranosa & 1 & 3,1 \\
Broncopneumonia & 5 & 15,6 \\
Subtotal & & \\
Não obstétricas & & \\
AlDS & 3 & 9,4 \\
Total & 32 & 100 \\
\hline
\end{tabular}

\section{Discussão}

A grande maioria das mulheres com morte materna no HCPA se encontrava na terceira década de vida, sendo este dado semelhante ao obtido em estudo realizado no Recife $^{2}$ onde a maioria das mortes se concentrou entre 20 e 29 anos. Também, esta década parece ser a mais prevalente na ocorrência das gestações, conforme estudo realizado em Caxias do $\mathrm{Sul}^{3}$, onde a média de idade das parturientes do Sistema Único de Saúde (SUS) foi de 24,7 anos. Também ressaltamos o fato de que $42 \%$ das mulheres que morreram não tinham realizado sequer uma consulta pré-natal. Este dado difere da população em geral, pois segundo dados da Secretaria de Saúde do Município de Porto Alegre, apenas 5\% das parturientes de Porto Alegre não realizam nenhuma consulta pré-natal, dado semelhante ao encontrado em Caxias do Sul, onde somente 4,6\% das parturientes do SUS não haviam realizado nenhuma consulta pré-natal ${ }^{3}$. Salientamos ainda o fato de $12,3 \%$ das mortes maternas estarem relacionadas ao aborto, dado semelhante ao obtido em Recife, onde 11,9\% correlacionou-se ao aborto. Note-se a importância desta ocorrência para a saúde pública, embora estes números possam ser ainda maiores, pois os índices de subnotificação para o aborto são, segundo Parpinelli et al. ${ }^{4}$, de cerca de $71 \%$. Cabe lembrar que a metodologia utilizada nessa pesquisa foi de analisar todos os casos de morte em mulheres em idade fértil, ou seja, se a paciente tivesse sido internada por outro diagnóstico que não o aborto, isso seria identificado.

O Ministério da Saúde do Brasil ${ }^{1}$ informou que no ano de 1998 ocorreram 3.144.547 nascidos vivos no país, com 2051 mortes maternas notificadas, o que espelha razão de morte materna oficial de 64,8:100.000. A estratificação destes números por região mostra: centro-oeste, 54,83:100.000; nordeste, 56,13:100.000; norte, 57,07:100.000; sudeste, 70,08:100.000; sul, 76,25:100.00. A análise destes números dá idéia da dimensão do sub-registro no país, uma vez que a aparente maior mortalidade nas regiões mais desenvolvidas (sul e sudeste) reflete apenas que nestas regiões a morte materna é melhor registrada e provavelmente mais estudada e que nos locais onde o problema é mais grave ele também é menos visivel. Na cidade de Porto Alegre, capital de um dos estados de melhores parâmetros socioeconômicos do país, a razão oficial de mortalidade materna no ano de 1999 foi de 62,8:100.000 ${ }^{5}$. Após a revisão de todos as declarações de óbito das mulheres em idade fértil, feita pelo Comitê Municipal de Mortalidade Materna, com busca ativa dos casos não notificados como de óbito materno, esta razão aumentou para 83,8:100.000 nascidos vivos. Já no HCPA, no período de 20 anos estudados, a razão de mortalidade, contabilizando somente os nascimentos realizados dentro do hospital, foi de 90:100.000, semelhante à razão encontrada na cidade no ano de 1999. Guando são adicionadas as pacientes que tiveram o parto em outros hospitais do estado, tendo sido encaminhadas já em estado crítico de saúde, a razão aumenta para 109:100.000.

As causas de morte materna mais prevalentes no HCPA não se modificaram nas duas últimas décadas, conforme estudo realizado previamente $^{6}$. As síndromes hipertensivas são a principal causa de mortalidade, o que também é encontrado em outras regiões do país ${ }^{7,8}$. Uma possível explicação para o fato é que, nos últimos dez anos, o HCPA diferenciou-se como centro de referência para gestações de alto risco e cuidados intensivos em gestantes, o que pode ter aumentado o risco geral da população hospitalar, com maior número de encaminhamentos de gestantes graves dos hospitais de menor complexidade. Esta explicação é reforçada pelo fato de que, nos últimos anos, as causas obstétricas diretas, como as mortes por HAS, tenham diminuído na cidade de Porto Alegre ${ }^{5}$.

Chama a atenção negativamente o número expressivo de mortes relacionadas à cesariana, principalmente os casos de infecções 
puerperais, justamente em um país onde as taxas médias de cesariana são sabidamente aumentadas. Constatamos risco de morte 10 vezes maior para as mulheres submetidas à cesariana quando comparadas às com parto vaginal. Cabe lembrar que no Hospital de Clínicas de Porto Alegre não há cesarianas marcadas eletivamente, a não ser os casos de 2 cesarianas prévias e de apresentação pélvica, sendo que a grande maioria destas cesarianas são de caráter de urgência. O risco maior de morte materna na cesariana é reconhecido por vários estudos nos mais diversos locais ${ }^{2}$. É de se salientar, entretanto, que mesmo nas mortes de pacientes com cesariana, nas quais o evento desencadeante do óbito relacionava-se com o ato operatório ou anestésico, todas as gestantes apresentavam fatores de risco, seja por doenças intercorrentes ou por baixo nível socioeconômico.

Em países desenvolvidos, como os Estados Unidos da América do Norte, o perfil epidemiológico da mortalidade materna aponta causas de mais difícil prevenção, como a tromboembolia e as causas obstétricas indiretas, como as mais prevalentes $^{9}$, demonstrando o resultado de efetivo controle dos casos de morte materna por infecção, hemorragia e hipertensão arterial.

É de salientar a dimensão da mortalidade no grupo de mulheres negras. Em Porto Alegre, apesar de somente $10,5 \%$ das mulheres serem negras, verificamos taxa de mortalidade de 18,9\% para este segmento da população feminina $\left(\chi^{2}=\right.$ $4,2 ; \mathrm{p}=0,04)$. Esta diferença também é verificada em países socialmente mais desenvolvidos como os Estados Unidos da América do Norte ${ }^{9}$. Quando é analisada a existência ou não de assistência prénatal ou o fato de a paciente ser ou não primigesta, com gestantes que não morreram, não foi constatada nenhuma diferença estatisticamente significativa $(\mathrm{p}>0,05)$.

Não é mais admissível que o processo fisiológico da reprodução humana esteja ligado a tamanho risco de mortalidade, principalmente em face dos grandes avanços sociais e da ciência e da tecnologia médica observados a partir das últimas décadas do século XX. A Organização Mundial de Saúde estimou, em 1990, que aproximadamente 585.000 mulheres morrem ao ano no mundo por complicações ligadas ao ciclo gravídicopuerperal, e que destas cerca de 95\% ocorreram nos países não desenvolvidos ${ }^{1}$. Na América Latina, a mortalidade materna é grave problema social e de saúde. Morrem anualmente nesta região em torno de 28.000 mulheres por complicações da gravidez, parto e puerpério ${ }^{1}$. Se todas as mulheres tivessem condições de vida e atenção à saúde semelhante às dos países mais desenvolvidos, poderiam ser evitadas $98 \%$ destas mortes, ou seja, 27.440 mortes maternas não aconteceriam a cada ano ${ }^{1}$.

Qualquer país que queira diminuir seus índices de mortalidade materna deve inicialmente considerar o tema da mortalidade materna como problema de direitos humanos e justiça social e promover a saúde feminina como sendo um investimento econômico e social altamente relevante. Deve-se também reconhecer que todas as gestações têm risco e que a divisão das gestantes como sendo de alto ou baixo risco de maneira nenhuma deve considerar estas últimas como isentas de risco e, portanto, relegadas a terem menos atenção pré e perinatal. Não menos importante é que toda e qualquer gestante tenha acesso facilitado aos diversos sistemas de saúde e sejam atendidas por pessoal especificamente treinado para dar assistência qualificada, principalmente durante o período mais crítico que é o do nascimento e o puerpério imediato. O treinamento dos prestadores de saúde da rede básica de atenção, no reconhecimento precoce dos fatores de risco, para encaminhar gestantes com potencial maior de mortalidade materna, antes de apresentarem as formas graves das enfermidades, é ponto-chave para combater a mortalidade materna. Deve-se ainda garantir que todos os partos ocorram dentro de hospitais e que haja sempre acesso a um banco de sangue. No trabalho de parto, a utilização de tecnologias simples como o partograma permite identificar e tratar precocemente os partos disfuncionais. Durante o pré-natal, devem ser diagnosticados e tratados todos os casos de infecções gênito-urinárias, e os casos de hipertensão arterial devem ser identificados ainda no seu início, para que estas gestantes possam ser encaminhadas aos centros terciários antes de apresentarem as formas graves da doença. Além disso, no sul do Brasil, fica bem evidente a necessidade de haver programas de educação médica continuada para combater o uso abusivo da cesariana e a implantação do planejamento familiar, visando evitar a ocorrência das gestações indesejada. Todas estas medidas devem ser permanentemente monitorizadas por meio do trabalho dos comitês hospitalares e regionais de vigilância da mortalidade materna.

O presente estudo, baseado na identificação permanente de todos os casos de morte materna que ocorreram no HCPA desde a inauguração de sua maternidade em 1980, pretende contribuir para que o problema da mortalidade materna no nosso meio seja melhor reconhecido e por conseqüência mais eficientemente combatido. 


\section{ABSTRACT}

Purpose: to analyze maternal death cases that occurred at the "Hospital de Clínicas de Porto Alegre", a reference university hospital for high-risk pregnancies in the state of Rio Grande do Sul, Brazil.

Methods: we carried out a retrospective study of medical records of 10- to 49-year-old women who died at the hospital between 1980 and 1999. Deaths related to pregnancy and puerperium were analyzed independently of the kind and duration of pregnancy. The causes were classified into direct obstetric, indirect obstetric and nonobstetric, according to their association with pregnancy, delivery and puerperium disorders.

Results: a total of 81 patients with a mean age of 28.5 years were studied. The maternal mortality rate was 109 per 100,000 live births. Direct obstetric causes made up $61.7 \%$ of deaths. Indirect causes made up $23.5 \%$ of deaths. Nonobstetric causes made up $15.0 \%$ of the total. Among direct obstetric causes, arterial hypertension (18.5\%), postcesarean infection (16\%), and septic abortion (12.3\%) were the most prevalent. The main maternal mortality events among indirect obstetric causes were cardiopathy (8.6\%), acute fatty liver disease (3.5\%), and systemic lupus erythematosus (2.5\%). Among the nonobstetric causes, malignant neoplasia (7.4\%) and AIDS (3.7\%) were the most important.

Conclusions: the prevailing causes of maternal death have not changed in the last two decades. The main cause is still hypertension. There is an expressive number of deaths related to cesarean section and infections. The high prevalence of direct obstetric causes shows the low maternal mortality prevention capacity of our health care system.

KEYWORDS: Maternal mortality. Hypertension. Postcesarean infection. High risk pregnancies. Septic abortion. Cesarean section.

\section{Referências}

1. Ministério da Saúde. Secretaria de Políticas de Saúde. Área Técnica da Saúde da Mulher. Manual dos Comitês de Mortalidade Materna. $2^{\text {a }}$ ed. Brasília; 2002.

2. Albuquerque RM, Cecatti JG, Hardy E, Faúndes A. Fatores sócio-demográficos e de assistência médica associados ao óbito materno. Rev Bras Ginecol Obstet 1998; 20:181-5.

3. Trevisan MR, De Lorenzi DRS, Araújo NM, Ësber K. Perfil da assistência pré-natal entre usuárias do Sistema Único de Saúde em Caxias do Sul. Rev Bras Ginecol Obstet 2002; 24:293-9.

4. Parpinelli MA, Faúndes A, Cecatti JG, et al. Subnotificação da mortalidade materna em Campinas: 1992 a 1994. Rev Bras Ginecol Obstet 2000; 22:27-32.

5. Prefeitura Municipal de Porto Alegre. Secretaria da Saúde e Meio Ambiente. Comitê de Mortalidade Materna. Porto Alegre; 1999.

6. Ramos JGL, Costa SM, Rosa Júnior A, Jiuliani RP, Corso MA. Mortalidade materna geral e por hipertensão arterial no estado do Rio Grande do Sul: uma análise de onze anos (1978-1988). Rev Bras Ginecol Obstet 1995; 17:123-9.

7. Laguardia KD, Rotholz MY, Belfort P. A 10-year review of maternal mortality in a municipal hospital in Rio de Janeiro: a cause for concern. Obstet Gynecol 1990; 75:27-32.

8. Sass N, Mattar R, Rocha NSC, Camano L. Coeficiente de mortalidade materna geral e decorrentes de hipertensão arterial na Escola Paulista de Medicina da Universidade Federal de São Paulo no período de 1983 à 1993. Rev Bras Ginecol Obstet 1995; 17:989-98.

9. Cunningham FG, MacDonald PC, Gant NF. Obstetrics in broad perspective. In: Cunningham FG, MacDonald PC, Gant NF, editors. Williams Obstetrics. 20 $0^{\text {th }}$ ed. Stanford: Appleton \& Lange; 1997. p.1-12. 